\title{
Powdery mildew resistance genes in barley varieties grown in Australia
}

\author{
Antonín Dreiseitl ${ }^{\mathrm{A}, \mathrm{C}}$ and Gregory Platz ${ }^{\mathrm{B}}$ \\ A Agricultural Research Institute Kromeriz Ltd, Havlíčkova 2787, CZ-767 01 Kroměřǐž, Czech Republic. \\ ${ }^{B}$ Hermitage Research Facility, 604 Yangan Road, Warwick, Qld 4370, Australia. \\ ${ }^{\mathrm{C}}$ Corresponding author. Email: dreiseitl.antonin@vukrom.cz
}

\begin{abstract}
Barley (Hordeum vulgare L.) is a major crop in Australia and powdery mildew (Blumeria graminis f. sp. hordei) is one of its most common diseases. Genes for resistance to powdery mildew were postulated for 86 Australian barley varieties and nine advanced breeding lines using 40 reference isolates of the pathogen. Fifty isolates collected in Australia in 2011 were used for additional tests of some varieties. In total, 22 known resistance genes [mlo, Mla1, MlaAl2, Mla3, Mla6, Mla 7, Mla8, Mla9, Mla12, Mla13, Mlat, Mlg, MlGa, Mlk1, MlLa, Mlra, Ml(Ab), Ml(Ch), Ml(Dr2), Ml(He2), Ml(Lo) and Ml(St)] were detected. The most frequent genes were Mla 8 and $M l g$ present in 43 and 34 varieties, respectively, while MlGa was found in 12 varieties. Each of the specific resistance genes Mla1, Mla3, Mla6, Mla9, Mla13, Ml(St) and the non-specific recessive gene mlo was found in one variety only. The varieties Maritime and Stirling appear to carry no specific resistance genes. Fifteen unknown resistances were detected. It is recommended that Australian barley breeding programs exploit European varieties possessing mlo to improve the resistance to powdery mildew in new varieties.
\end{abstract}

Additional keywords: Blumeria graminis f. sp. hordei, gene postulation, Hordeum vulgare, pathogen isolates, pedigree analysis, resistance spectra.

Received 17 April 2012, accepted 21 August 2012, published online 2 November 2012

\section{Introduction}

Barley (Hordeum vulgare L.) is a major crop worldwide and powdery mildew caused by the biotrophic airborne fungus Blumeria graminis (DC.) E. O. Speer, f. sp. hordei emend. É. J. Marchal (anamorph Oidium monilioides Link), hereafter designated $B g h$, is one of its most common diseases. The largest areas and highest concentration of barley production are in Europe where high-input management practices of both spring and winter forms prevail. Favourable climatic conditions for the development of $B g h$ and the availability of host tissues all year round result in barley infection every year (Dreiseitl $2011 a$ ) causing losses in grain yield and quality. In Europe, Bgh is effectively controlled by exploitation of genetic resistance (Jørgensen 1994), which is a cheap and environmentally friendly means of control. Widespread diversity of potential sources of resistance to $B g h$ has been found (Dreiseitl and Dinoor 2004) and several new specific resistances have recently been detected in cultivated varieties (Dreiseitl 2011b, 2011c, 2011d, 2011e). However, specific resistances have not proven durable and breeders have opted to use a fully effective recessive gene of non-specific resistance mlo (Jørgensen 1992). Therefore, over the last two decades, spring barleys, possessing mlo have predominated.

Barley is the second most important cereal crop in Australia and is grown on $\sim 4.4$ million ha with an average annual production of
7.52 million $\mathrm{t}$ (2001-10) (ABARES 2011). Domestic consumption is $\sim 2.85$ million $t$ leaving $60-65 \%$ of the grain available for export. Australia supplies almost one-third of the world's malting barley trade and $\sim 20 \%$ of the world's feed trade (www.barleyaustralia. com.au/IndustryInformation/barley/tabid/56/Default.aspx). The crop is grown from southern Queensland to Western Australia, including Tasmania, in environments that range from subtropical to Mediterranean. Varieties are spring types sown in late autumnearly winter. Foliar diseases are a major constraint to barley production with powdery mildew occurring in all regions. Its occurrence over wide areas has been estimated to cause annual losses of $\$ 39$ million with the potential to reduce production by up to $\$ 103$ million (Murray and Brennan 2010). The average yield of barley is low (1.74t/ha; 2001-10; ABARES 2011), therefore the most economical way to control $B g h$ is to develop and grow genetically resistant varieties. In Australia several programs have been successful in breeding cereals for rust resistance (Park 2008). Similar progress can be achieved in breeding barley for resistance to $B g h$.

Currently, powdery mildew resistance of barley varieties in the field depends mainly on the presence of major genes represented by genes of specific resistance or by the gene mlo. Individual genes of specific resistances differ substantially in their effectiveness against the pathogen population comprising both virulent and avirulent pathotypes. Therefore, knowledge 
of the genetic background of varietal resistance is important for characterising host-pathogen interactions (Czembor and Czembor 2002; Silvar et al. 2011), for improving methods of resistance breeding (Shtaya et al. 2007; Bogacki et al. 2008; Repková et al. 2009; Hickey et al. 2012) and for analysing the components of non-specific resistance, which is considered to be more durable than specific resistance (Lillemo et al. 2010; Goyeau and Lannou 2011).

Disease resistance genes are postulated on the basis of specific interactions of the host with pathogen isolates of known virulences (Dreiseitl and Steffenson 2000; Kolmer 2003; Zhang et al. 2010). The number of resistances, and especially their combinations, that can be postulated depends on the availability of appropriate biological material, i.e. standard host genotypes representing all possible specific resistances as well as accurately characterised pathogen isolates covering virulences or avirulences to these resistances.

Australian breeding programs have attempted to breed barley for resistance to $B g h$ with varying levels of success. This has resulted from a lack of information on both resistances in varieties and virulences in the pathogen population. Knowledge of the resistances in commercial varieties and advanced breeding lines is key to their effective exploitation for genetic control. Therefore, the main goals of this study were: $(i)$ to test a set of barley varieties to a wide range of reference $B g h$ isolates possessing broad spectra of virulences or avirulences and define the resistance spectra of these varieties; (ii) to compare the resistance spectra of tested varieties with those of standard lines possessing known resistance genes, and on this basis, postulate resistance genes in these varieties, and (iii) to compare the results obtained with the pedigrees of the varieties tested.

\section{Materials and methods}

\section{Plant materials}

Eighty-five barley varieties registered in Australia from 1967 to 2011, an old variety Cape, nine advanced breeding lines and 234 individual plant progenies from mixed genotypes were tested. Seed of all the varieties was provided by the Australian Winter Cereals Collection or by Australian barley breeders while seed of the individual plant progenies grown from the original barley samples was provided by Agricultural Research Institute Kromeriz, Czech Republic. The varieties studied and their pedigrees are listed in Table 1.

\section{Pathogen isolates}

Forty selected reference isolates of Bgh held in the pathogen collection at the Agricultural Research Institute Kromeriz were used for response tests. Pathotype designation was derived from their virulence patterns corresponding to 12 near-isogenic lines (Pallas) (Kølster et al. 1986), in coded triplets (Limpert and Müller 1994) in the order of their $M l$ virulence $(V)$ genes: $a 1$, a3, a6; a7, a9, a12; a13, k1, La; g, at and (Ru2). Before inoculation, each isolate was purified, verified for the correct virulence phenotype on standard barley lines and increased on leaf segments of a mildew susceptible line B-3213. Fifty isolates collected in Australia in 2011 were used for additional tests on some varieties.

\section{Testing procedure}

The experiments were carried out at the Agricultural Research Institute Kromeriz, Czech Republic. About 40-50 untreated seeds of each variety were sown in two pots $(80 \mathrm{~mm}$ diameter) filled with a gardening peat substrate and kept in a mildew-proof greenhouse under natural daylight. Leaf segments $20 \mathrm{~mm}$ long were cut from the central part of healthy fully-expanded primary leaves when second leaves were emerging. For testing, three leaf segments of each variety were placed with the adaxial side uppermost in a Petri dish on water agar $(0.8 \%)$ containing benzimidazole $\left(40 \mathrm{mg}^{-\mathrm{L}}\right)-\mathrm{a}$ leaf senescence inhibitor. For each isolate, a Petri dish with leaf segments was placed at the bottom of a metal inoculation tower (Limpert 1987) and inoculated by blowing spores collected from infected leaf segments of the line B-3213 over the Petri dish at an inoculum density of $\sim 8$ conidia $\mathrm{mm}^{-2}$. The dishes with inoculated leaf segments were incubated at $18 \pm 2^{\circ} \mathrm{C}$ under artificial light (cool-white fluorescent lamps providing $12 \mathrm{~h}$ light at $\left.30 \pm 5 \mu \mathrm{mol} \mathrm{m}^{-2} \mathrm{~s}^{-1}\right)$.

\section{Evaluation}

Eight days after inoculation, reaction types $(\mathrm{RT}=$ phenotype of variety $\times$ isolate interaction) on the central part of the adaxial side of leaf segments were scored on a 0-4 scale (Torp et al. 1978). Each variety was tested on a minimum of two replications. If there were noticeable differences in RT between replications, additional tests were conducted. A set of $40 \mathrm{RT}$ provided a resistance spectrum (RS) of each variety tested with reference isolates and a set of $50 \mathrm{RT}$ provided a RS of each variety tested with Australian isolates. Based on the gene-for-gene model (Flor 1971), the resistance in each variety was postulated by comparing the RS with previously determined RS of standard barley varieties possessing known resistance genes.

\section{Results}

Thirty-eight varieties exhibited homogeneous reactions to all $B g h$ pathotypes used and their genes for resistance to powdery mildew were postulated based on results from testing plants that had emerged from the original seed. Plants of another 57 varieties exhibited different reactions to single $B g h$ isolates and were deemed heterogenous. Of these, 20 varieties (Barque, Bass, Baudin, Binalong, Buloke, Clipper, Cowabbie, Doolup, Galleon, Grout, Hamelin, Lockyer, Mackay, Molloy, Morrell, Tilga, Wyalong, Yagan, Yambla and Yerong) were characterised by low numbers of plants with different responses that obviously arose from mechanical admixtures of other varieties. After eliminating the minority components displaying atypical reactions, RS were obtained that allowed us to postulate the resistances of these varieties based on results of testing the plants emerged from the true seed.

In the remaining 37 heterogeneous varieties we found higher numbers of plants with different RT that did not allow us to derive $\mathrm{RS}$ and determine the resistances of these varieties. Therefore, individual kernels of the original seed of these varieties were sown and 3-15 plants were harvested individually from each variety. In addition to the original set of 95 varieties, 234 progenies of individually harvested plants were tested. Progenies of 14 of these heterogeneous varieties (Cape, Dash, Finniss, 
Flagship, Galaxy, Gilbert, Kaputar, Keel, Maritime, Schooner, Ulandra, Unicorn, WABAR2452 and WABAR2478) exhibited identical resistances. Component lines carrying different resistances to powdery mildew were detected in progenies of each of the other 23 heterogeneous varieties (Brindabella, Cantala, Commander, Corvette, Dhow, Dictator, Fairview, Fitzgerald, Forrest, Franklin, Lindwall, Macumba, Moby, Namoi, Onslow, Tallon, Torrens, Tulla, Urambie, Windich, Yarra, VB0611 and WB259).

All tests done with 40 reference isolates on 95 varieties and their single plant progenies resulted in 49 RS. Each variety was given a spectrum number and its resistance gene(s) postulated (Table 1). Fourteen selected isolates were sufficient to separate all RS. The RS and the postulated resistance genes are listed in Table 2. Additional tests were done with 50 Australian isolates on 32 varieties. These tests on five selected varieties and some of their single plant progenies resulted in seven RS. Twelve isolates were selected to show these RS (Table 3). Seven other lines were found in five heterogeneous varieties (Brindabella, Dhow, Fairview, Moby and Urambie) when tested with Australian isolates.

In total, 22 known resistance genes [ $m l o$, Mla1, MlaAl2, Mla3, Mla6, Mla 7, Mla8, Mla9, Mla12, Mla13, Mlat, Mlg, MlGa, Mlk1, MlLa, Mlra, Ml(Ab), Ml(Ch), Ml(Dr2), Ml(He2), Ml(Lo) and Ml $(S t)]$ were detected. The most frequent genes were Mla 8 and $M l g$ found in 43 and 34 varieties, respectively. The gene $M l G a$ was found in 12 varieties, the gene MlLa in 15 and the gene Mlkl in seven varieties. Each of the specific resistance genes Mla1, Mla3, Mla6, Mla9, Mla13 and Ml(St) was found in one variety only. In Maritime and Stirling no specific resistance was found and in Galaxy a non-specific recessive gene mlo was detected. In 11 varieties (Brindabella, Cowabbie, Dhow, Dictator, Fairview, Fitzgerald, Milby, Moby, Namoi, Urambie and WB259) 15 unknown RS were found. Twenty-three varieties exhibited heterogeneity for mildew resistance where their component lines were shown to possess different resistance genes. Fourteen of these heterogeneous varieties were composed of two lines; five varieties of three lines; Corvette and Yarra of four lines; five lines were detected in Moby and six lines in Fitzgerald.

\section{Discussion}

Major genes conferring resistance to powdery mildew can be found in almost all current European barley varieties (Brown and Jørgensen 1991; Dreiseitl and Križanová 2012). In this study at least one major gene was found in 93 of the 95 varieties tested. This is a result of exploiting more and more resistance genes and an increasing ability to postulate those genes. In 19 varieties tested herein only one of the genes $M l a 8, M l(C h)$ or $M l(L o)$ was found. Detection of these resistance genes requires the use of rare avirulent isolates (Dreiseitl 2011e). There are no such pathotypes with avirulence to the three resistance genes present in current pathogen populations or, if so, their occurrence is very rare. Therefore, the practical importance of these genes in the field is zero. In some laboratories that use a similar method to identify resistance genes, but do not use the rare avirulent isolates as used here, it could be concluded that 21 and not two of the varieties tested do not carry a specific gene for powdery mildew resistance.
Resistance genes for which there are only a few avirulent isolates for postulation, cannot be reliably detected if combined with other genes. For example, in varieties possessing $M l g$, the gene Mla 8 cannot be detected because it can be found only with the avirulent isolate 1044, which is also avirulent to $\mathrm{Mlg}$. Likewise, $M l(C h)$ can only be detected using isolate 1044 and therefore, it cannot be detected if either Mlg or Mla 8 are also present. The genes Mla 8 and Mlg were the most frequent in the set examined. Mlg was certainly detected in the varieties tested yet Mla 8 is certain to be present in some varieties additional to those in which it was found. Mla8 is not only likely to be in many varieties carrying $M l g$, but also in others possessing different resistance genes. For example a phenotypic response of RT0 after inoculation with isolate 1044 does not allow the detection of Mla 8 as the isolate gives a similar response on many other $M l$ resistance genes.

It is known that six of the resistance genes detected (Mla1, Mla6, Mla7, Mla12, Mla13 and Mlg) are closely linked to other genes in coupling (MlaAl2, Mla14, MlaNo3 or MlaMu2, MlaEm2, MlaRu3 or MlaRu4 and MlCP, respectively) (Jørgensen 1994), and therefore, these genes can be assumed to be present in the corresponding varieties. To find these 'additional' genes was not the aim of this study because their contribution to resistance of varieties is relatively small. Furthermore, there are only a few known isolates that could confirm their presence. Despite using a large number of reference isolates (40) for resistance tests, only one of them (1044) indicated the presence of MlaAl2 in Vertess. The presence of the other 'additional' genes can be assumed, but cannot be confirmed with the isolates used.

The postulation of mlo is based on two observations: $(i)$ the absence of susceptible infection responses (RT 4 or 3-4) and (ii) the presence of a small number of fully or almost fully developed colonies of $B g h$ (generally $<5 \%$ of the colonies on a susceptible control). This phenotype [RT0(4) (Jensen et al. 1992), more frequently RT0(3) in our present and previous tests] is specific for mlo. However, nearly all current barley varieties, including those with mlo, simultaneously carry one or more genes for specific resistance. These specific resistance genes prevent the development of the phenotype, typical for mlo in certain variety $\times$ isolate interactions. Typical RT0(3) with sparse pathogen colonies may not express even at a lower inoculum density, and particularly in tests on leaf segments. Some varieties are composed of two or more lines carrying different genes for specific resistance. For these reasons, RT0(3) can be determined in varieties with mlo only in a limited number of variety/isolate interactions; therefore detection of $m l o$ is difficult and even impossible where highly effective specific resistance genes are present. This renders postulation of specific-resistance genes in varieties with mlo difficult and necessitates a larger number of replications.

Thirty-two varieties were tested with the standard 40 reference isolates and with an additional 50 Australian isolates. In five heterogeneous varieties, the additional tests provided new information on their resistances. It is documented by seven RS given in Table 3 . The new information was obtained by testing single plant progenies of these varieties that had not been tested with the reference isolates. For example, when Dhow was tested with the reference isolates two lines were detected. One carried $M l g, M l k 1$ and the other Mlg, Mlkl, MlU. However, in the 
Table 1. Eighty-six barley varieties registered in Australia, nine advanced breeding lines, their pedigrees and postulated $M l$ genes for resistance to powdery mildew

\begin{tabular}{|c|c|c|c|c|c|}
\hline Cultivar & $\begin{array}{l}\text { Original } \\
\text { designation }\end{array}$ & $\begin{array}{l}\text { Year of } \\
\text { registration }\end{array}$ & Pedigree & $\begin{array}{l}\text { Resistance } \\
\text { spectrum }\end{array}$ & $\begin{array}{l}M l \text { resistance } \\
\text { gene(s) }\end{array}$ \\
\hline Arapiles & Barley 568,8727 & 1993 & $\begin{array}{l}\text { Noyep/Proctor//CI3576/Union/4/Kenia/ } \\
\text { 3/Research/ }\end{array}$ & 14 & $a 8$ \\
\hline Bandulla & В 6513 & 1981 & Prior/Lenta//Noyep/Lenta & 14 & $a 8$ \\
\hline Barque & WI2868 & 1997 & Triumph/Galleon & 34 & $G a$ \\
\hline Bass & WABAR2315 & 2011 & B28719/Alexis & 14 & $a 8$ \\
\hline Brindabella & OR $385-1-2$ & 1993 & $\begin{array}{l}\text { Weeah/CI7115//HCB27/3/JadarII/4/ } \\
\text { Cantala }\end{array}$ & $14+\mathrm{A} 1$ & $a 8+U, a 8$ \\
\hline Buloke & VB0105 & 2005 & Franklin/2*VB9104(Europa/7IBON148) & 12 & $a 7, L a$ \\
\hline Bussel & A11 & 1967 & Prior/Ymer & 14 & $a 8$ \\
\hline Cantala & B 74043 & 1981 & Kenia/Erectoides 16 & $14+20$ & $a 8+a 8, L a$ \\
\hline Cape & CIho 1026 & Early 1900 s & Unknown - South Africa & 16 & $a 8, a t$ \\
\hline Capstan & - & - & WI2395/3/Schooner//Chariot/Chebec & - & - \\
\hline Chebec & WI2737 & 1992 & Orge Martin/2*Clipper(86)//Schooner & 14 & $a 8$ \\
\hline Clipper & WI2095/10 & 1968 & Proctor/PriorA & 19 & $a 8, k 1$ \\
\hline Commander & WI3416-1572 & 2004 & Keel/Sloop//Galaxy & $31+33$ & $g, G a+g, L a$ \\
\hline Corvette & $\begin{array}{l}\text { WI2355 } \\
-\end{array}$ & $\begin{array}{l}1976 \\
-\end{array}$ & $\begin{array}{l}\text { Bonus/CI3576 } \\
-\end{array}$ & $\begin{array}{l}14+17+27+ \\
31\end{array}$ & $\begin{array}{l}a 8+a 8, G a+g+ \\
g, G a\end{array}$ \\
\hline Cowabbie & WB236 & 2002 & $\begin{array}{l}\text { AB6/2*Franklin//Rubin/Skiff (AB6 is } \\
\text { Hordeum spontaneum } \mathrm{CP} 171283 / \\
4 * \text { Clipper) }\end{array}$ & 46 & $U, a 8$ \\
\hline \multirow[t]{2}{*}{ Dash } & NFC 902/909 & 1995 & Chad/Joline//Cask & 13 & $a 7, k 1, L a$ \\
\hline & - & - & - & - & - \\
\hline Dhow & WI3102 & 2002 & $\begin{array}{l}\text { WI2808((Clipper*CPI-18197)/ } \\
\text { 14*2EBYT23))// }\end{array}$ & $\mathrm{A} 2+32+49$ & $g+g, k 1+$ \\
\hline Doolup & - & - & 4/Clipper/Tenn65-117) & - & - \\
\hline Fairview & - & 2007 & Alexis/H86004-37 (IMC breeder's line) & $25+26+\mathrm{A} 3$ & $a 13+a 13, g+U$ \\
\hline Finniss & WI3930 & 2009 & Galleon//Skiff/CIMMYT42002 & 3 & $(\mathrm{Ch}),(\mathrm{He} 2)$ \\
\hline \multirow[t]{3}{*}{ Fitzgerald } & WABAR2030 & 1997 & Onslow/Tas85-466(Shannon/Triumph) & $14+15+27+$ & $a 8+a 8,(A b)+\mathrm{g}+$ \\
\hline & - & - & - & $33+42+47$ & $g, L a+U, a 7+U$ \\
\hline & - & - & - & - & as \\
\hline \multirow[t]{2}{*}{ Fitzroy } & VB9926 & 2005 & $\begin{array}{l}\text { WI2808 (Clipper*CPI-18197)/ } \\
\text { 14*2EBYT23) }\end{array}$ & 32 & $g, k l$ \\
\hline & - & - & /Alexis & - & - \\
\hline \multirow[t]{2}{*}{ Flagship } & WI3408 & 2005 & $\begin{array}{l}\text { Chieftain/Barque//Manley/VB9104 } \\
\text { (Europa/ }\end{array}$ & 31 & $g, G a$ \\
\hline & - & - & 7IBON148) & - & - \\
\hline Fleet & WI3804 & 2006 & Multan/Keel//Barque & 34 & $G a$ \\
\hline Forrest & $68 \mathrm{~S} 17-11-8$ & 1980 & Atlas57//A16(Prior/Ymer) & $27+29$ & $g+g, a t$ \\
\hline Franklin & Barley $485,85-83$ & 1989 & Shannon/Triumph & $14+20$ & $a 8+a 8, L a$ \\
\hline Gairdner & WABAR2034 & 1997 & Onslow/Tas83-587(Shannon/Triumph) & 27 & $g$ \\
\hline Galaxy & Osprey & 1993 & Robin/24719DB & 37 & mlo \\
\hline Galleon & WI2231B & 1981 & Clipper/Hiproly//3*Proctor/CI3576 & 34 & $G a$ \\
\hline Gilbert & $M x-2-45 B$ & 1993 & Reselection of Koru (Armelle//Lud/Luke) & 33 & $g, L a$ \\
\hline Grimmett & Bus*Zep 166 & 1982 & Bussel/Zephyr & 27 & $g$ \\
\hline
\end{tabular}


Table 1. (continued)

\begin{tabular}{|c|c|c|c|c|c|}
\hline Cultivar & $\begin{array}{l}\text { Original } \\
\text { designation }\end{array}$ & $\begin{array}{l}\text { Year of } \\
\text { registration }\end{array}$ & Pedigree & $\begin{array}{l}\text { Resistance } \\
\text { spectrum }\end{array}$ & $\begin{array}{l}M l \text { resistance } \\
\text { gene(s) }\end{array}$ \\
\hline Grout & NRB01001 & 2005 & Cameo/Arupo & 21 & $a 9, g$ \\
\hline Hamelin & WABAR2104 & 2001 & Stirling/Harrington & 27 & $g$ \\
\hline Hannan & WABAR2321 & 2007 & WABAR2023//Windich/Morex & 18 & $a 8,(\mathrm{He} 2)$ \\
\hline Harrington & Barley 1935 (Canada) & 1981 & Klages/3/Gazelle/Betzes/Centennial & 27 & $g$ \\
\hline Hindmarsh & VB0324 & 2007 & Dash/VB9409(O’Connor/WI2723) & 20 & $a 8, L a$ \\
\hline Kaputar & Barley 577, Arupo 'S' & 1993 & $\begin{array}{l}\text { 5604/1025/3/Emir/Shabet//CM67/4/ } \\
\text { F3Bulk HIP }\end{array}$ & 9 & $a 6, g$ \\
\hline Keel & WI2976 & 1999 & CPI18197/Clipper//WI2645(Mari/CM67) & 27 & $g$ \\
\hline Lindwall & $\mathrm{T} / \mathrm{G} 121$ & 1997 & Triumph/Grimmett & $14+15$ & $a 8+a 8,(A b)$ \\
\hline Lockyer & WABAR2288 & 2007 & $\begin{array}{l}\text { Tantangara/VB9104((Europa/ } \\
\text { 7IBON148) }\end{array}$ & 20 & $a 8, L a$ \\
\hline Mackay & CK85 & 2002 & Cameo/Koru & 33 & $g, L a$ \\
\hline Macquarie & $\mathrm{T} 1677$ & 2008 & Gairdner//Alexis/Gairdner & 27 & $g$ \\
\hline Macumba & WI3693 & 2009 & Azhul/Barque//Keel & $1+27+34$ & none $+g+G a$ \\
\hline Malebo & WWB858 & 1981 & $\begin{array}{l}\text { Selection from CPI11083(Palladium } \\
\text { WWB18) }\end{array}$ & 14 & a8 \\
\hline \multirow[t]{2}{*}{ Maritime } & WI3297 & 2004 & $\begin{array}{l}\text { Dampier/A14//Kristina/3/Clipper/M11/ } \\
\text { Dampier/ }\end{array}$ & 1 & none \\
\hline & - & - & 14//Kristina/3/Dampier/A14//Union & - & - \\
\hline Milby & WB238 & 2002 & AB6/2*Franklin//Earubin/Skiff & 45 & $U, a 8$ \\
\hline Moby & PGB01 & 2009 & Selection from Dictator & $\begin{array}{l}\mathrm{A} 4+\mathrm{A} 5+ \\
\mathrm{A} 6+39+48\end{array}$ & none $+(H e 2)+\mathrm{U}+\mathrm{U}+U, g$ \\
\hline \multirow[t]{2}{*}{ Molloy } & WABAR0519; & 1996 & $\begin{array}{l}\text { Golden Promise/WI2395(WARI2-38)/4/ } \\
\text { 72S:267 }\end{array}$ & 17 & $a 8, G a$ \\
\hline & 83S:519 & - & $(\mathrm{XBVT} 210) / 3 / 66 \mathrm{~S} 08-4$ & - & - \\
\hline \multirow[t]{2}{*}{ Moondyne } & $745 / 312$ & 1987 & $\begin{array}{l}\text { Dampier//A14(Prior/Ymer)/3/Kristina } \\
\quad(70 \text { S20-20) }\end{array}$ & 14 & $a 8$ \\
\hline & - & - & $/ 4 / 73 \mathrm{~S} 13$ & - & - \\
\hline \multirow[t]{2}{*}{ Morrell } & S2SN:513; 82S953-5 & 1993 & $\begin{array}{l}\text { WUM221/P23822 (81S806)/5/Forrest } \\
(81 \mathrm{~S} 719) / 4 /\end{array}$ & 27 & $g$ \\
\hline & - & - & $\begin{array}{l}\text { Psaknon(80S564)/Dampier//M19 } \\
\text { (76T111)/3/Zephyr }\end{array}$ & - & - \\
\hline Mundah & $835-514$ & 1996 & O'Connor/Yagan & 14 & $a 8$ \\
\hline \multirow[t]{2}{*}{ Namoi } & Calidad MIS74; & 1993 & Sultan/Nackta//RM1508/Godiva & $2+43$ & $(C h)+U, a 7, g, k 1$ \\
\hline & AUS400533 & - & - & - & - \\
\hline \multirow[t]{3}{*}{ Navigator } & WI4262 & 2011 & $\begin{array}{l}\text { Chieftain/VB9624(Skiff/WI2738(Orge } \\
\text { Martin }\end{array}$ & 24 & $a 12, g$ \\
\hline & - & - & $\begin{array}{l}\text { /2*Clipper//Schooner)/4/Keel/3/Sahara/ } \\
\quad \text { WI2723// }\end{array}$ & - & - \\
\hline & - & - & Chebec/5/BX98A;080-375 & _- & - \\
\hline \multirow[t]{2}{*}{ O'Connor } & $72 \mathrm{~S} / 221$ & 1983 & Proctor/CI3576/3/(XBVT212)Atlas57// & 14 & a8 \\
\hline & - & - & A14(Prior/Ymer) & - & - \\
\hline Onslow & $77 \mathrm{~S}: 399 ; 77 \mathrm{~S} 167-7-26$ & 1989 & Forrest/Aapo & $20+27$ & $a 8, L a+g$ \\
\hline Oxford & - & 2009 & Tavern/Chime & 38 & $(S t)$ \\
\hline Picola & 860453 & 1996 & 75031/Elgina & 14 & $a 8$ \\
\hline Roe & WABAR2310 & 2007 & Doolup//Windich/Morex & 18 & $a 8,(H e 2)$ \\
\hline Shepherd & NRB03470 & 2008 & Reselection of Baronesse & 8 & $a 3$ \\
\hline Schooner & WI2468 & 1983 & Proctor/PriorA//Proctor/CI3576 & 17 & $a 8, G a$ \\
\hline \multirow[t]{2}{*}{ Skiff } & WI2584 & 1988 & $\begin{array}{l}\text { Abed Deba/3/Proctor/CI3576//CPI18197/ } \\
\text { Beka/4/ }\end{array}$ & 2 & $(C h)$ \\
\hline & - & - & Clipper/Diamant//Proctor/CI3576 & - & - \\
\hline \multirow[t]{2}{*}{ Sloop } & WI2875-22 & 1997 & $\begin{array}{l}\text { WI2468/Norbert//Golden Promise/ } \\
\text { WI2395/3/ }\end{array}$ & 17 & $a 8, G a$ \\
\hline & - & - & Schooner & - & - \\
\hline Stirling & 70 S21-53-4 & 1981 & Dampier//(A14)Prior/Ymer/3/Piroline & 1 & none \\
\hline \multirow[t]{2}{*}{ Tallon } & TMP*GMT306/13 & 1991 & Triumph/Grimmett & $10+11$ & $a 7,(A b)+$ \\
\hline & - & - & - & - & $a 7,(A b), g$ \\
\hline \multirow[t]{2}{*}{ Tantangara } & WB198; A\%1055 & 1995 & AB6/Skiff(AB6 is Hordeum spontaneum & 2 & $(C h)$ \\
\hline & - & - & CP171283/4*Clipper) & - & - \\
\hline
\end{tabular}


Table 1. (continued)

\begin{tabular}{|c|c|c|c|c|c|}
\hline Cultivar & $\begin{array}{l}\text { Original } \\
\text { designation }\end{array}$ & $\begin{array}{l}\text { Year of } \\
\text { registration }\end{array}$ & Pedigree & $\begin{array}{l}\text { Resistance } \\
\text { spectrum }\end{array}$ & $\begin{array}{l}M l \text { resistance } \\
\text { gene(s) }\end{array}$ \\
\hline Tilga & 8913 & 1997 & Forrest/Cantala & 14 & $a 8$ \\
\hline Torrens & WI3107 & 2001 & Galleon/Cimmyt42002 & $14+27$ & $a 8+g$ \\
\hline Triumph & Trumph & 1985 & Diamant/ST1402964-6 & 10 & $a 7,(A b)$ \\
\hline Tulla & WB230 & 2002 & Skiff/FM437 & $14+36$ & $a 8+(L o)$ \\
\hline Ulandra & WU3076 & 1987 & Warboys/Alpha & 30 & $g,(D r 2)$ \\
\hline Unicorn & Kinukei 21 & 1997 & $54 \mathrm{C} 25 / 51 \mathrm{C} 38$ & 14 & $a 8$ \\
\hline Urambie & WB234 & 2006 & Yagan/2*Ulandra & $14+44+\mathrm{A} 7$ & $a 8+U, a 8+U, g$ \\
\hline Vertess & T98-189 & 2005 & Franklin/Cooper & 7 & $a 1, a A l 2, L a$ \\
\hline \multirow[t]{2}{*}{ Vlamingh } & WABAR2175 & 2006 & $\begin{array}{l}\text { WABAR0570(72-0785/Tokak/5/ } \\
\text { Dampier/A14//Kna }\end{array}$ & 14 & $a 8$ \\
\hline & - & - & $\begin{array}{l}\text { /3/Sutter/4/Atlas57/A16//Clipper/Delisa)/ } \\
\text { TR118 }\end{array}$ & - & - \\
\hline \multirow[t]{2}{*}{ Waranga } & 81507; Vic10 & 1987 & $\begin{array}{l}\text { PlumageArcher/3/Prior/Lenta/2/ } \\
\text { Research/Lenta/4/ }\end{array}$ & 35 & $k 1$ \\
\hline & - & - & Clipper & - & - \\
\hline Weeah & W4059 & 1968 & Prior/Research & 14 & $a 8$ \\
\hline \multirow[t]{2}{*}{ Windich } & $75 S: 329$ & 1988 & $\begin{array}{l}\text { Atlas57//(A16)Prior/Ymer(68S17-75)/3/ } \\
\quad \text { (B6729) }\end{array}$ & $14+27$ & $a 8+g$ \\
\hline & - & - & Prior/Lenta//Noyep/Lenta & - & - \\
\hline Wyalong & WB190R & 1998 & Schooner/Stirling & 34 & $G a$ \\
\hline Yagan & IB/286:WUM143 & 1988 & Unknown CIMMYT & 6 & $(C h), r a$ \\
\hline Yambla & WB220 & 1998 & Skiff/FM437 & 14 & as \\
\hline \multirow[t]{3}{*}{ Yarra } & VB0021 & 2005 & $\begin{array}{l}\text { VB9018(Clipper/Galleon)/Alexis// } \\
\text { VB9104 }\end{array}$ & $1+3+4+5$ & none $+(\mathrm{Ch}),(\mathrm{He} 2)$ \\
\hline & - & - & ((Europa/7IBON148) & - & $+(\mathrm{Ch}),(\mathrm{He} 2), \mathrm{La}$ \\
\hline & - & - & - & - & $+(C h), L a$ \\
\hline Yerong & $\mathrm{WB} 135=\mathrm{GR} 84 \% 4293$ & 1991 & M22/Malebo & 2 & $(C h)$ \\
\hline Zephyr & - & 2001 & Heine2149/Carlsberg & 27 & $g$ \\
\hline ND19119-5 & - & - & ND15403-3/ND15368//ND16453 & 19 & $a 8, k 1$ \\
\hline NRB06059 & - & - & $\begin{array}{l}\text { Mackay*2/WI3214 (Triumph/Galleon// } \\
\text { Harrington) }\end{array}$ & 33 & $g, L a$ \\
\hline \multirow[t]{2}{*}{ VB0611 } & - & - & $\begin{array}{l}\text { VB9729((WI2869(Triumph/Galleon)/ } \\
\text { Alexis)) }\end{array}$ & $15+28$ & $a 8,(A b)+g,(A b)$ \\
\hline & - & - & $\begin{array}{l}\text { /19IBON097//VB0025(VB9107/Alexis// } \\
\text { VB9104) }\end{array}$ & - & - \\
\hline \multirow[t]{3}{*}{ VB0613 } & - & - & $\begin{array}{l}\text { VB9733((Fergie/VB9107(Europa/ } \\
\text { 7IBON148)) }\end{array}$ & 20 & $a 8, L a$ \\
\hline & - & - & $\begin{array}{l}\text { /VB9729((WI2869(Triumph/Grimmett)/ } \\
\text { Alexis))// }\end{array}$ & - & - \\
\hline & - & - & VB0025(VB9107/Alexis//VB9104) & - & - \\
\hline WABAR2385 & - & - & Chebec/Harrington-b60//2*Harrington & 27 & $g$ \\
\hline WABAR2452 & - & - & Yagan/Natasha//TR118 & 23 & $a 12,(A b)$ \\
\hline WABAR2478 & - & - & W92\%794/4*Baudin & 2 & $(C h)$ \\
\hline WB259 & - & - & Skiff/FM437//Franklin & $1+41$ & none $+U$ \\
\hline WI2291 & - & - & CI3576/Union//Union & 27 & $g$ \\
\hline
\end{tabular}

$\mathrm{U}=$ Unknown.

additional tests using the Australian isolates, single plant progenies were found as carrying the genes $\mathrm{Mlg}$ or $\mathrm{Mlg}, \mathrm{Mlkl}$. The line carrying $M l g$ should have been easily detected using the reference isolates but it was not present. This indicates that if more single plant progenies had been tested, more component lines may have been detected in some varieties. For example, in Macumba we found three lines: one possessing $M l G a$, one with $M l g$ and a line without any resistance gene (none). However, we can assume the presence of a fourth possible line in Macumba possessing the combination of $M l G a$ and $M l g$ which should be found if more progenies are tested.
Most current varieties were developed by crossing parents with different resistances. Segregation of genes in the resultant progeny is a source of heterogeneity in varieties. However, heterogeneity is also often caused by mechanical admixtures with other varieties. To postulate resistance genes in heterogeneous varieties, homogeneous component lines or a specific method of testing are usually required (Dreiseitl 2011f). If the number of homogeneous samples tested is low, the probability of detecting all lines constituting the given variety decreases. Conversely, the higher the number tested, the greater the probability of detecting random mechanical admixtures with 
Table 2. Forty-nine resistance spectra found in 86 barley varieties registered in Australia and nine advanced breeding lines after inoculation with 14 reference isolates of Blumeria graminis f. sp. hordei

\begin{tabular}{|c|c|c|c|c|c|c|c|c|c|c|c|c|c|c|c|}
\hline \multirow{2}{*}{$\begin{array}{l}\text { Resistance } \\
\text { spectrum }\end{array}$} & \multirow{2}{*}{$\begin{array}{l}M l \text { resistance } \\
\text { genes }\end{array}$} & \multicolumn{14}{|c|}{ Isolate of the $B . g . h o r d e i^{\mathrm{A}}$} \\
\hline & & 0023 & 0061 & 0235 & 1002 & 1044 & 2567 & 3777 & 4114 & 4517 & 4773 & 5774 & 6577 & 7467 & 7555 \\
\hline 1 & none & 4 & 4 & 4 & 4 & 4 & 4 & 4 & 4 & 4 & 4 & 4 & 4 & 4 & 4 \\
\hline 2 & $(C h)$ & 4 & 4 & 4 & 4 & 2 & 4 & 4 & 4 & 4 & 4 & 4 & 4 & 4 & 4 \\
\hline 3 & $(\mathrm{Ch}),(\mathrm{He} 2)$ & 4 & $2-3$ & 4 & 4 & 2 & 4 & 4 & 4 & 4 & 4 & 4 & 4 & 4 & 4 \\
\hline 4 & $(\mathrm{Ch}),(\mathrm{He} 2), \mathrm{La}$ & $2-3$ & $2-3$ & $2-3$ & $2-3$ & 2 & 4 & 4 & $2-3$ & $2-3$ & 4 & 4 & 4 & 4 & 4 \\
\hline 5 & $(C h), L a$ & $2-3$ & 4 & $2-3$ & $2-3$ & 2 & 4 & 4 & $2-3$ & $2-3$ & 4 & 4 & 4 & 4 & 4 \\
\hline 6 & $(C h), r a$ & 4 & 4 & 4 & 1 & 2 & 4 & 4 & 4 & 4 & 4 & 4 & 4 & 4 & 4 \\
\hline 7 & $a 1, a A l 2, L a$ & 0 & 0 & 0 & $2-3$ & $1-2$ & 0 & 4 & 0 & 0 & 0 & 4 & 0 & 4 & 4 \\
\hline 8 & $a 3$ & 1 & 1 & 1 & 1 & 1 & 4 & 4 & 1 & 1 & 1 & 1 & 4 & 4 & 4 \\
\hline 9 & $a 6, g$ & 0 & 0 & 0 & 0 & 0 & 0 & 0 & 0 & 4 & 4 & 0 & 4 & 4 & 4 \\
\hline 10 & $a 7,(A b)$ & 0 & 0 & 0 & 0 & 0 & 4 & $2-3$ & $2-3$ & 4 & 4 & 4 & 4 & 0 & 4 \\
\hline 11 & $a 7,(A b), g$ & 0 & 0 & 0 & 0 & 0 & 4 & $2-3$ & 0 & 4 & 4 & 0 & 4 & 0 & 4 \\
\hline 12 & $a 7, L a$ & 0 & 0 & 0 & 0 & 0 & 4 & 4 & $2-3$ & $2-3$ & 4 & 4 & 4 & 0 & 4 \\
\hline 13 & $a 7, k 1, L a$ & 0 & 0 & 0 & 0 & 0 & 4 & 4 & $1-2$ & $1-2$ & 4 & 4 & 4 & 0 & $1-2$ \\
\hline 14 & $a 8$ & 4 & 4 & 4 & 4 & 0 & 4 & 4 & 4 & 4 & 4 & 4 & 4 & 4 & 4 \\
\hline 15 & $a 8,(A b)$ & 4 & 4 & $2-3$ & $2-3$ & 0 & 4 & $2-3$ & $2-3$ & 4 & 4 & 4 & 4 & 4 & 4 \\
\hline 16 & $a 8, a t$ & 4 & 2 & 2 & 4 & 0 & 4 & 4 & 2 & 4 & 4 & 2 & 4 & 4 & 2 \\
\hline 17 & $a 8, G a$ & 2 & 2 & 2 & 4 & 0 & 4 & 4 & 2 & 2 & 2 & 2 & 2 & 4 & 2 \\
\hline 18 & $a 8,(\mathrm{He} 2)$ & 4 & $2-3$ & 4 & 4 & 0 & 4 & 4 & 4 & 4 & 4 & 4 & 4 & 4 & 4 \\
\hline 19 & $a 8, k 1$ & 4 & 4 & 4 & $1-2$ & 0 & 4 & 4 & $1-2$ & $1-2$ & 4 & 4 & 4 & 4 & $1-2$ \\
\hline 20 & $a 8, L a$ & $2-3$ & 4 & $2-3$ & $2-3$ & 0 & 4 & 4 & $2-3$ & $2-3$ & 4 & 4 & 4 & 4 & 4 \\
\hline 21 & $a 9, g$ & 0 & 0 & 4 & 0 & 0 & 4 & 4 & 0 & 4 & 4 & 0 & 0 & 0 & 4 \\
\hline 22 & $a 12$ & 0 & 0 & 0 & 0 & 0 & 4 & 4 & 0 & 4 & 4 & 4 & 4 & 4 & 4 \\
\hline 23 & $a 12,(A b)$ & 0 & 0 & 0 & 0 & 0 & 4 & $2-3$ & 0 & 4 & 4 & 4 & 4 & 4 & 4 \\
\hline 24 & $a 12, g$ & 0 & 0 & 0 & 0 & 0 & 4 & 4 & 0 & 4 & 4 & 0 & 4 & 4 & 4 \\
\hline 24 & $a 12, g$ & 0 & 0 & 0 & 0 & 0 & 4 & 4 & 0 & 4 & 4 & 0 & 4 & 4 & 4 \\
\hline 25 & $a 13$ & 0 & 0 & 4 & 0 & 0 & 0 & 4 & 4 & 4 & 4 & 4 & 4 & 0 & 4 \\
\hline 26 & $a 13, g$ & 0 & 0 & 4 & 0 & 0 & 0 & 4 & 0 & 4 & 4 & 0 & 4 & 0 & 4 \\
\hline 27 & $g$ & 4 & 4 & 4 & 0 & 0 & 4 & 4 & 0 & 4 & 4 & 0 & 4 & 4 & 4 \\
\hline 28 & $g,(A b)$ & 4 & 4 & $2-3$ & 0 & 0 & 4 & $2-3$ & 0 & 4 & 4 & 0 & 4 & 4 & 4 \\
\hline 29 & $g$, at & 4 & 2 & 2 & 0 & 0 & 4 & 4 & 0 & 4 & 4 & 0 & 4 & 4 & 2 \\
\hline 30 & $g,(D r 2)$ & 2 & 4 & 4 & 0 & 0 & 4 & 4 & 0 & 4 & 4 & 0 & 4 & 4 & 4 \\
\hline 31 & $g, G a$ & 2 & 2 & 2 & 0 & 0 & 4 & 4 & 0 & 2 & 2 & 2 & 2 & 4 & 2 \\
\hline 32 & $g, k l$ & 4 & 4 & 4 & 0 & 0 & 4 & 4 & 0 & $1-2$ & 4 & 0 & 4 & 4 & $1-2$ \\
\hline 33 & $g, L a$ & $2-3$ & 4 & $2-3$ & 0 & 0 & 4 & 4 & 0 & $2-3$ & 4 & 0 & 4 & 4 & 4 \\
\hline 34 & $G a$ & 2 & 2 & 2 & 4 & 2 & 4 & 4 & 2 & 2 & 4 & 2 & 2 & 4 & 2 \\
\hline 35 & $k l$ & 4 & 4 & 4 & $1-2$ & $1-2$ & 4 & 4 & $1-2$ & $1-2$ & 4 & 4 & 4 & 4 & $1-2$ \\
\hline 36 & $(L o)$ & 4 & 4 & 4 & 4 & 0 & 4 & 4 & 4 & 4 & 4 & 4 & 4 & 0 & 4 \\
\hline 37 & mlo & 0 & 0 & 0 & 0 & 0 & $0(3)$ & $0(3)$ & 0 & 0 & $0(3)$ & 0 & $0(3)$ & $0(3)$ & $0(3)$ \\
\hline 38 & $(S t)$ & 0 & 0 & 0 & 0 & 0 & 4 & 0 & 0 & 4 & 4 & 4 & 4 & 0 & 0 \\
\hline 39 & $U$ & 4 & 4 & 4 & 4 & 2 & 4 & 4 & 4 & 4 & 2 & 2 & 4 & 4 & 4 \\
\hline 40 & $U$ & 4 & 4 & 2 & 4 & 2 & 4 & 4 & 4 & 4 & 4 & 4 & 4 & 4 & 4 \\
\hline 41 & $U$ & 4 & 4 & 2 & 2 & 2 & 4 & 2 & 2 & 4 & 4 & 4 & 4 & 4 & 4 \\
\hline 42 & $U, a 7$ & 0 & 0 & 0 & 0 & 0 & 2 & 2 & 2 & 2 & 2 & 2 & 4 & 0 & 4 \\
\hline 43 & $U, a 7, g, k 1$ & 0 & 0 & 0 & 0 & 0 & 2 & 2 & 0 & 2 & 2 & 0 & 4 & 0 & 2 \\
\hline 44 & $U, a 8$ & 4 & 4 & 4 & 4 & 0 & 4 & 4 & 4 & 2 & 4 & 4 & 4 & 4 & 2 \\
\hline 45 & $U, a 8$ & 4 & 2 & 2 & 2 & 0 & 4 & 4 & 4 & 4 & 4 & 4 & 4 & 2 & 4 \\
\hline 46 & $U, a 8$ & 4 & 2 & 2 & 2 & 0 & 4 & 2 & 2 & 4 & 4 & 4 & 4 & 4 & 4 \\
\hline 47 & $U, a 8$ & 4 & 4 & 2 & 4 & 0 & 4 & 4 & 4 & 4 & 4 & 4 & 4 & 4 & 4 \\
\hline 48 & $U, g$ & 4 & 4 & 4 & 0 & 0 & 4 & 4 & 0 & 4 & 2 & 0 & 4 & 4 & 4 \\
\hline 49 & $U, g, k l$ & 2 & 2 & 4 & 0 & 0 & 4 & 4 & 0 & 2 & 2 & 0 & 2 & 2 & 2 \\
\hline
\end{tabular}

${ }^{\mathrm{A}}$ Virulence codes according to Limpert and Müller (1994).

other varieties, which may have been erroneously considered as lines of that variety. The sampling and testing of heterogeneous varieties are much more laborious and the results apply only at that point in time because the heterogeneity of the variety can change over time due to random or purposeful selection of constituent lines.
Corvette was derived from the cross Bonus $\times$ CI3576. Bonus carries Mla8 (Jørgensen and Jensen 1983) and CI3576 has Mlg (Brückner 1964) and MlGa (Hossain and Sparrow 1991a, 1991b). It is easy to combine all three genes and therefore, eight potential genotypes can be developed after crossing these varieties. Only six of the eight potential resistance combinations could 
Table 3. Seven resistance spectra found in five barley varieties after inoculation with 12 Australian isolates of Blumeria graminis f. sp. hordei

\begin{tabular}{|c|c|c|c|c|c|c|c|c|c|c|c|c|c|c|}
\hline \multirow{2}{*}{$\begin{array}{l}\text { Resistance } \\
\text { spectrum }\end{array}$} & \multirow[t]{2}{*}{ Variety } & \multirow{2}{*}{$\begin{array}{l}M l \text { resistance } \\
\text { genes }\end{array}$} & \multicolumn{12}{|c|}{ Isolate of $B$. g. hordei collected in $2011^{\mathrm{A}}$} \\
\hline & & & $501 Q$ & $506 \mathrm{Q}$ & $519 \mathrm{~S}$ & $520 \mathrm{~S}$ & $534 \mathrm{~V}$ & $561 \mathrm{~V}$ & $618 \mathrm{~W}$ & $648 \mathrm{~W}$ & $655 \mathrm{~N}$ & $682 \mathrm{~N}$ & $600 \mathrm{~T}$ & $692 \mathrm{~T}$ \\
\hline A1 & Brindabella & $U$ & 4 & 4 & 4 & 4 & 4 & 4 & $2-3$ & $2-3$ & $2-3$ & 4 & 4 & 4 \\
\hline A3 & Fairview - selection & $U$ & $2-3$ & $2-3$ & $2-3$ & $2-3$ & $2-3$ & $2-3$ & $2-3$ & $2-3$ & $2-3$ & $2-3$ & $2-3$ & $2-3$ \\
\hline A4 & Moby - selection & none & 4 & 4 & 4 & 4 & 4 & 4 & 4 & 4 & 4 & 4 & 4 & 4 \\
\hline A5 & Moby - selection & $(\mathrm{He} 2)$ & 4 & 4 & 4 & $2-3$ & $2-3$ & $2-3$ & $2-3$ & $2-3$ & 4 & $2-3$ & $2-3$ & $2-3$ \\
\hline A6 & Moby - selection & $U$ & 1 & 1 & 1 & 1 & 1 & 1 & 1 & 1 & 1 & 1 & 1 & 1 \\
\hline
\end{tabular}

${ }^{\mathrm{A}}$ Letter in isolate designation defines its origin $(\mathrm{Q}=$ Queensland, $\mathrm{S}=$ South Australia, $\mathrm{V}=$ Victoria, $\mathrm{W}=$ Western Australia, $\mathrm{N}=$ New South Wales and $\mathrm{T}=$ Tasmania)

be detected with the isolates used because combinations of Mla 8 , $M l g$ and $M l a 8, M l g, M l G a$ cannot be distinguished from the single $M l g$ and the combination $M l g, M l G a$, respectively, (see above). Tests on progeny of six single plant selections revealed four lines with various combinations of genes. Thus, Corvette is a good example of a heterogeneous (multiline) variety resulting from segregation of parental resistance genes in progenies after crossing.

A contrasting example is Gilbert, which is a selection from the English variety Koru. In the original sample of Gilbert, a high proportion of plants with different resistances was found. In three progenies tested, however, only lines with an identical combination of genes $\mathrm{MlLa}$ and $\mathrm{Mlg}$, carried by Koru (Jensen et al. 1992), were detected. The heterogeneity of the original sample of Gilbert was obviously caused by mechanical admixtures of varieties with other resistances and none of these 'other resistances' was present in the three single plant progenies. This could also be the case for variety Namoi, in which the combination of genes $M l a 7, M l g, M l k l$ and $M l U$ was found in five lines, whereas the gene $\mathrm{Ml}(\mathrm{Ch})$ was detected in only one line. Such segregation of genes is highly unlikely and the plant progeny with the gene $M l(C h)$ can therefore be assumed to be a mechanical admixture of another variety.

Galleon had a small number of plants with different resistances that were obviously a mechanical admixture of another variety. Originally, Galleon was composed of two lines, one of which possessed a single gene and the other two dominant genes including an unknown gene for powdery mildew resistance (Hossain and Sparrow 1991a). This second line was not detected in the sample received; so Galleon is an example of the change in heterogeneity in a variety over time.

The resistance gene in Galleon was designated $M l G a$ referring to the name of this variety (Hossain and Sparrow 1991a). In this study, the gene $M l G a$ was also detected in two of the four lines found in Corvette, which was registered 5 years before Galleon, and also in Capstan and Schooner. Pedigrees of all four varieties contain the Egyptian variety CI3576, which is the donor of $M l G a$. This gene was also detected in eight other varieties (Barque, Commander, Flagship, Fleet, Macumba, Molloy, Sloop and Wyalong). Galleon is the donor of MlGa in Barque, Fleet, Macumba, and Flagship, and Schooner for Sloop and Wyalong. The donor of MlGa in Molloy is not apparent from the pedigree. CI3576 is also in the pedigrees of Arapiles, O'Connor, Skiff and WI2291, but MlGa was not found in them. Although the gene $M l G a$ was derived from the Egyptian variety CI3576 it can be referred to as 'Australian' because it was initially described by Australian authors (Hossain and Sparrow 1991a, 1991b). It was used in Australian commercial varieties only; yet in terms of understanding host-pathogen interactions it is the most important specific resistance gene emanating from Australia.

Based on pedigrees, 33 of the 95 varieties tested herein are parents of at least 1 of the 59 descendant varieties tested. The most frequent parents were Clipper, Skiff and Triumph, which appear in the pedigrees of 15,10 and 9 descendant varieties, respectively. Mlk1 possessed by Clipper is obviously derived from Prior A. Out of 15 varieties with parent Clipper, the gene $M l k l$ was found in three only (Dhow, Fitzroy and Warranga). Mlkl in Dash is derived from Joline (Jensen et al. 1992), whereas its origin in Namoi and ND19119-5 is not apparent from their pedigrees.

In five genotypes (Commander, Gilbert, Fitzgerald, Mackay and NRB06059), a combination of the genes MlLa and Mlg were detected. Commander has Keel $(M l g)$ in its pedigree; however, $M I L a$ could only be derived from Galaxy. If this is the case then in Galaxy MlLa is masked by the epistatic effect of the gene mlo. Gilbert was selected from Koru, which carries an identical combination of resistance genes (Jensen et al. 1992); Mackay originated from the cross Cameo/Koru and NRB06059 has Mackay in its pedigree. The pedigree of Fitzgerald contains Onslow, in which the two genes mentioned were found in our tests; however, in different lines. It cannot be excluded that the line of Onslow used for the cross from which Fitzgerald was derived, could carry both $M l L a$ and $M l g$, although it was not detected here.

The gene Mlat found in Cape and in a line of Forrest has been successfully exploited in a few commercial varieties, especially those bred in the Czech Republic and Slovakia in the 1980s (Dreiseitl and Jørgensen 2000). Mlat is often present in North African (Morocco) landraces (Czembor 2000). Cape is a very old six-row South African variety with unknown pedigree, registered in Australia at the beginning of the last century. It is more likely a landrace than a variety selected after crossing. Forrest was registered in 1980 and its Mlat resistance was probably derived from Atlas 57 (Wiberg 1974).

Using both reference and Australian isolates, eleven varieties produced 15 RSs that differed from those of known resistances. Most of the RS contain RT2 and higher. The phenotype of these resistances is more prone to environmental influences and subjective evaluation can be an additional source of error; therefore, obtaining accurate spectra of the component resistances 
requires increased replication. In heterogeneous varieties, single plant progenies exhibited different resistances. For example, six lines were detected in Fitzgerald and the RS obtained could not always be confirmed by the limited number of repeated tests. Therefore, the RS may not reflect all of the different resistances, but those that could be identified as known resistances or combinations of known resistances.

Almost all the known genes conferring resistance to barley varieties are specific and can be overcome by a simple mutation in the pathogen population and subsequent reproduction of individuals with the new virulence. The specific resistance, though it can be initially very effective, is usually overcome within a few years of widespread cultivation of such varieties (Dreiseitl 2011b, 2011c). Non-specific resistances should be durable (Brown et al. 1997). The gene mlo demonstrates a unique mode of action (Jørgensen 1993) combining the advantages of specific (monogenic inheritance) and nonspecific resistances. Gene mlo exhibits almost full resistance to barley powdery mildew and it is considered as non-host resistance (Zellerhoff et al. 2010).

The gene mlo is employed in varieties of spring barley only. The first commercial variety carrying mlo was Atem, registered in 1979. Since then, the area sown to varieties with mlo has been increasing (Jørgensen 1992) and such varieties predominate among newly registered varieties in Europe. Over the period it has been deployed, mlo has delivered a huge economic and environmental benefit mainly to European farmers; not only reducing losses to $B g h$ but also avoiding the need to apply thousands of $t$ of fungicides. Only one variety (Galaxy) was found to carry mlo among the 95 varieties examined here.

Environmental conditions in most Australian barley-growing regions differ from those prevailing in Europe. Nevertheless, some European varieties such as Baronesse and Koru were reselected and released as varieties in Australia under the names Shepherd and Gilbert, respectively. There has been some reluctance by breeders in Australia to use mlo due to its perceived sensitivity to physiological leaf spotting and heat stress. Currrently, there are scores of European varieties possessing mlo and thus it can be expected that types suitable for Australian conditions could be selected from them. These varieties provide superior sources of powdery mildew resistance and should be useful for further exploitation by the Australian barley breeding programs.

\section{Acknowledgements}

The excellent technical assistance of Mrs Dagmar Krejčírová is greatly appreciated. The article was prepared within project no. RO-0211-3021 supported by the Ministry of Agriculture of the Czech Republic.

\section{References}

ABARES (2011) 'Agricultural commodity statistics 2011.' (Department of Agriculture, Fisheries and Forestry: Canberra)

Bogacki P, Oldach KH, Williams KJ (2008) Expression profiling and mapping of defence response genes associated with the barley-Pyrenophora teres incompatible interaction. Molecular Plant Pathology 9, 645-660. doi:10.1111/j.1364-3703.2008.00485.x
Brown JKM, Foster EM, O'Hara BO (1997) Adaptation of powdery mildew populations to cereal varieties in relation to durable and non-durable resistance. In 'The gene-for-gene relationship in plant-parasite interactions'. (Eds IR Crute, EB Holub, JJ Burdon) pp. 119-138. (CAB International: Oxon, UK)

Brown JKM, Jørgensen JH (1991) A catalogue of mildew resistance genes in European barley varieties. In 'Integrated control of cereal mildews: virulence and their change'. (Ed. JH Jørgensen) pp. 263-286. (Risø National Laboratory: Roskilde, Denmark)

Brückner F (1964) Powdery mildew (Erysiphe graminis DC.) on barley. V. The resistance of barley varieties to physiological races of Erysiphe graminis DC. detected in Czechoslovakia and the possibility to use it in breeding for resistance. Rostlinna Vyroba 10, 395-408.

Czembor JH (2000) Resistance to powdery mildew in populations of barley landraces from Moroco. Genetic Resources and Crop Evolution 47, 439-449. doi:10.1023/A:1008732919426

Czembor JH, Czembor HJ (2002) Selections from barley landraces collected in Libya as new sources of effective resistance to powdery mildew (Blumeria graminis f. sp. hordei). Rostlinna Vyroba 48, 217-223.

Dreiseitl A (2011a) Differences in powdery mildew epidemics in spring and winter barley based on 30-year variety trials. Annals of Applied Biology 159, 49-57. doi:10.1111/j.1744-7348.2011.00474.x

Dreiseitl A (2011b) Resistance of 'Roxana' to powdery mildew and its presence in some European spring barley cultivars. Plant Breeding 130, 419-422. doi:10.1111/j.1439-0523.2010.01786.x

Dreiseitl A (2011c) Resistance of 'Laverda' to powdery mildew and its presence in some winter barley cultivars. Cereal Research Communications 39, 569-576. doi:10.1556/CRC.2011.002

Dreiseitl A (2011d) Presence of the newly designated powdery mildew resistance Landi in some winter barley cultivars. Czech Journal of Genetics and Plant Breeding 47, 64-68.

Dreiseitl A (2011e) Dissimilarity of barley powdery mildew resistances Heils Hanna and Lomerit. Czech Journal of Genetics and Plant Breeding 47, 95-100.

Dreiseitl A (2011f) Postulation of resistance genes to barley diseases in heterogeneous varieties. Biologia 66, 762-767. doi:10.2478/s11756011-0080-8

Dreiseitl A, Dinoor A (2004) Phenotypic diversity of barley powdery mildew resistance sources. Genetic Resources and Crop Evolution 51, 251-257. doi:10.1023/B:GRES.0000024010.12369.b3

Dreiseitl A, Jørgensen JH (2000) Powdery mildew resistance in Czech and Slovak barley cultivars. Plant Breeding 119, 203-209. doi:10.1046/ j.1439-0523.2000.00473.x

Dreiseitl A, Križanová K (2012) Powdery mildew resistance genes in spring barley cultivars registered in Slovakia from 2000 to 2010. Cereal Research Communications 40, 494-501. doi:10.1556/CRC.2012.0008

Dreiseitl A, Steffenson BJ (2000) Postulation of leaf rust resistance genes in Czech and Slovak barley cultivars and breeding lines. Plant Breeding 119, 211-214. doi:10.1046/j.1439-0523.2000.00495.x

Flor HH (1971) Current status of the gene-for-gene concept. Annual Review of Phytopathology 9, 275-296. doi:10.1146/annurev.py.09.090171. 001423

Goyeau H, Lannou C (2011) Specific resistance to leaf rust expressed at the seedling stage in cultivars grown in France from 1983 to 2007. Euphytica 178, 45-62. doi:10.1007/s10681-010-0261-5

Hickey LT, Lawson W, Platz GJ, Fowler RA, Arief V, Dieters M, German S, Fletcher S, Park RF, Singh D, Pereyra S, Franckowiak J (2012) Mapping quantitative trait loci for partial resistance to powdery mildew in an Australian barley population. Crop Science 52, 1021-1032. doi:10.2135/cropsci2011.10.0535

Hossain MA, Sparrow DHB (1991a) Resistance to powdery mildew (Erysiphe graminis f. sp. hordei) in the barley cultivar Galleon. I. Relationship with known genes for resistance. Euphytica 52, 1-9. 
Hossain MA, Sparrow DHB (1991b) Resistance to powdery mildew (Erysiphe graminis f. sp. hordei) in the barley cultivar Galleon. II. Chromosomal location and linkage with hordein protein genes. Euphytica 52, 11-17.

Jensen HP, Christensen E, Jørgensen JH (1992) Powdery mildew resistance genes in 127 Northwest European spring barley varieties. Plant Breeding 108, 210-228. doi:10.1111/j.1439-0523.1992.tb00122.x

Jørgensen JH (1992) Discovery, characterization and exploitation of Mlo powdery mildew resistance in barley. Euphytica 63, 141-152. doi:10.1007/BF00023919

Jørgensen JH (1993) Durability of resistance in the pathosystem: barleypowdery mildew. In 'Durability of disease resistance'. (Eds TH Jacobs, JE Parlevliet) pp. 159-176. (Kluwer Academic Publishers: Dordrecht, The Netherlands)

Jørgensen JH (1994) Genetics of powdery mildew resistance in barley. Critical Reviews in Plant Sciences 13, 97-119. doi:10.1080/0735268 9409701910

Jørgensen JH, Jensen HP (1983) Powdery mildew resistance gene Mla8 (Reg1h8) in Northwest European spring barley varieties. Barley Genetics Newsletter 13, 51-52.

Kolmer JA (2003) Postulation of leaf rust resistance genes in selected soft red winter wheats. Crop Science 43, 1266-1274. doi:10.2135/cropsci2003. 1266

Kølster P, Munk L, Stølen O, Løhde J (1986) Near-isogenic barley lines with genes for resistance to powdery mildew. Crop Science 26, 903-907. doi:10.2135/cropsci1986.0011183X002600050014x

Lillemo M, Skinnes H, Brown JKM (2010) Race specific resistance to powdery mildew in Scandinavian wheat cultivars, breeding lines and introduced genotypes with partial resistance. Plant Breeding 129, 297-303. doi:10.1111/j.1439-0523.2009.01691.x

Limpert E (1987) Frequencies of virulence and fungicide resistance in the European barley mildew population in 1985. Journal of Phytopathology 119, 298-311. doi:10.1111/j.1439-0434.1987.tb04401.x
Limpert E, Müller K (1994) Designation of pathotypes of plant pathogens. Journal of Phytopathology 140, 346-358. doi:10.1111/j.1439-0434. 1994.tb00617.x

Murray GM, Brennan JP (2010) Estimating disease losses to the Australian barley industry. Australasian Plant Pathology 39, 85-96. doi:10.1071/ AP09064

Park RF (2008) Breeding cereals for rust resistance in Australia. Plant Pathology 57, 591-602. doi:10.1111/j.1365-3059.2008.01836.x

Řepková J, Dreiseitl A, Lízal P (2009) A new CAPS marker for selection of a barley powdery mildew resistance gene in the Mla locus. Cereal Research Communications 37, 93-99. doi:10.1556/CRC.37.2009.1.11

Shtaya MJY, Sillero JC, Flath K, Pickering R, Rubiales D (2007) The resistance to leaf rust and powdery mildew of recombinant lines of barley (Hordeum vulgare L.) derived from $H$. vulgare $\times H$. bulbosum crosses. Plant Breeding 126, 259-267. doi:10.1111/j.1439-0523.2007. 01328.x

Silvar C, Flath K, Kopanke D, Gracia MP, Lasa JM, Casas AM, Igartua E, Ordon F (2011) Analysis of powdery mildew resistance in the Spanish barley core collection. Plant Breeding 130, 195-202. doi:10.1111/j.14390523.2010.01843.x

Torp J, Jensen HP, Jørgensen JH (1978) 'Powdery mildew resistance genes in 106 Northwest European spring barley varieties.' (Royal Veterinary and Agricultural University: Copenhagen, Denmark)

Wiberg A (1974) Sources of resistance to powdery mildew in barley. Hereditas 78, 1-40. doi:10.1111/j.1601-5223.1974.tb01426.x

Zellerhoff N, Himmelbach A, Dong W, Bieri S, Schaffrath U, Schweizer P (2010) Nonhost resistance of barley to different fungal pathogens is associated with largely distinct, quantitative transcriptional responses. Plant Physiology 152, 2053-2066. doi:10.1104/pp.109.151829

Zhang J, Wellings CR, McIntosh RA, Park RF (2010) Seedling resistances to rust diseases in international triticale germplasm. Crop \& Pasture Science 61, 1036-1048. doi:10.1071/CP10252 